\title{
LETTERS
}

\section{Rapid cloning of high-affinity human monoclonal antibodies against influenza virus}

\author{
Jens Wrammert ${ }^{1 *}$, Kenneth Smith ${ }^{3 *}$, Joe Miller ${ }^{1}$, William A. Langley ${ }^{1}$, Kenneth Kokko ${ }^{2}$, Christian Larsen ${ }^{2}$, \\ Nai-Ying Zheng ${ }^{3}$, Israel Mays ${ }^{3}$, Lori Garman ${ }^{3}$, Christina Helms ${ }^{3}$, Judith James ${ }^{4,6,7}$, Gillian M. Air ${ }^{8}$, J. Donald Capra ${ }^{5,9}$, \\ Rafi Ahmed ${ }^{1} \&$ Patrick C. Wilson ${ }^{3,6,9}$
}

Pre-existing neutralizing antibody provides the first line of defence against pathogens in general. For influenza virus, annual vaccinations are given to maintain protective levels of antibody against the currently circulating strains. Here we report that after booster vaccination there was a rapid and robust influenzaspecific $\mathrm{IgG}^{+}$antibody-secreting plasma cell (ASC) response that peaked at approximately day 7 and accounted for up to $6 \%$ of peripheral blood B cells. These ASCs could be distinguished from influenza-specific $\mathrm{IgG}^{+}$memory $\mathrm{B}$ cells that peaked 14-21 days after vaccination and averaged $1 \%$ of all B cells. Importantly, as much as $80 \%$ of ASCs purified at the peak of the response were influenza specific. This ASC response was characterized by a highly restricted $\mathrm{B}$-cell receptor (BCR) repertoire that in some donors was dominated by only a few B-cell clones. This pauciclonal response, however, showed extensive intraclonal diversification from accumulated somatic mutations. We used the immunoglobulin variable regions isolated from sorted single ASCs to produce over 50 human monoclonal antibodies (mAbs) that bound to the three influenza vaccine strains with high affinity. This strategy demonstrates that we can generate multiple highaffinity $m A b s$ from humans within a month after vaccination. The panel of influenza-virus-specific human mAbs allowed us to address the issue of original antigenic sin (OAS): the phenomenon where the induced antibody shows higher affinity to a previously encountered influenza virus strain compared with the virus strain present in the vaccine ${ }^{1}$. However, we found that most of the influenza-virus-specific mAbs showed the highest affinity for the current vaccine strain. Thus, OAS does not seem to be a common occurrence in normal, healthy adults receiving influenza vaccination.

Influenza causes 36,000 deaths annually in the USA alone, and the influenza pandemic of 1918 caused an estimated 50 million deaths worldwide ${ }^{2}$. Outbreaks of avian influenza infections in human populations that caused substantially higher mortality rates foresee the possibility of another deadly pandemic ${ }^{3}$. The challenge of influenza has long been to design vaccines that induce long-lasting immunity against a pathogen that rapidly alters its appearance to the immune system by mutating (antigenic drift) and exchanging (antigenic shift) its components. Antibodies play a key role in protection against influenza infection ${ }^{4-7}$. However, the underlying B-cell response leading to the rapid production of ASCs that secrete antibodies is only beginning to be understood ${ }^{8-12}$. Critically, we do not yet know if B-cell memory can provide sufficient protection early in the response to counteract variant strains of influenza or if the response is dominated by antibodies previously generated against divergent viruses in an OAS fashion. Finally, of profound clinical significance is the possibility that the early ASC response observed after immunization can be exploited to generate therapeutic or diagnostic mAbs rapidly to emerging influenza virus strains, or to any immunizing antigen.

To determine the dynamics and magnitude of the human antiinfluenza response, we analysed the frequency of ASCs and memory B cells in a time course after vaccination. The ASC response was quite transient, peaking at approximately day 7 and returning to barely detectable levels by day 14 after vaccination (Fig. 1a, b). The frequency of influenza-specific ASCs averaged 6.4\% (about 2,500 ASCs per millilitre of blood) at day 7, and accounted for up to $16 \%$ of all B cells (range for ten donors: 1.1-16\%, Fig. 1b). Also, most of these ASCs were generated during the vaccination response as almost all ASCs expressed the protein bound by the Ki- 67 antibody, indicating recent proliferation, and most expressed homogenously high levels of human leukocyte antigen DR (HLA-DR) ${ }^{13}$ (Fig. 1c). Importantly, analysis of IgG-secreting ASCs isolated by cell sorting at day 7 post-immunization demonstrated that most were influenzavaccine-specific (ranging from $20 \%$ to $85 \%$, average $70 \%$; Fig. 1d). The ASCs were mainly IgG positive, with minor components of IgAand IgM-positive cells (data not shown), suggesting an origin from the memory B-cell compartment. The memory B-cell response was also quantified ${ }^{14}$. Increasing from low levels before vaccination, influenza-specific memory B cells peaked a week after the ASC response at $14-28$ days after vaccination and averaged $8.2 \%$ of the $\mathrm{IgG}^{+}$ memory B cells, or about $1 \%$ of all B cells (Fig. 1e). We conclude that influenza vaccination results in a massive burst of $\mathrm{IgG}^{+} \mathrm{ASCs}$ that are predominantly influenza reactive and peak at approximately day 7 post-immunization.

The rapid accumulation of ASCs suggests that the response could be highly clonal in nature, limiting the early influenza response. Some clonal activation of ASCs occurs after tetanus vaccination ${ }^{12}$. We therefore analysed the immunoglobulin repertoire breadth (that is, the variable genes and junctional diversity) of the influenza-specific ASCs. Influenza vaccination caused a surprisingly pauci-clonal response, with some donors being dominated by the progeny of only a few expanded B-cell clones (Fig. 2a and Supplementary Fig. 1a).

\footnotetext{
${ }^{1}$ Emory Vaccine Center and Department of Microbiology and Immunology, Emory University School of Medicine, Atlanta, Georgia 30322 , USA. ${ }^{2}$ Emory Transplant Center and Department of Surgery, Emory University School of Medicine, Atlanta, Georgia 30322, USA. ${ }^{3}$ Immunobiology and Cancer Research Program, The Oklahoma Medical Research

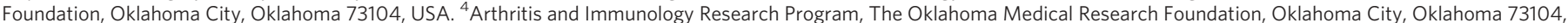

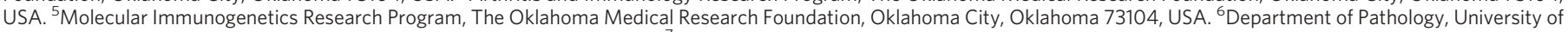

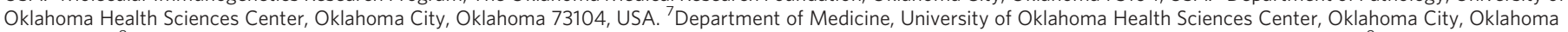
73104, USA. ${ }^{8}$ Department of Biochemistry and Molecular Biology, University of Oklahoma Health Sciences Center, Oklahoma City, Oklahoma 73104, USA. ${ }^{9}$ Department of Microbiology and Immunology, University of Oklahoma Health Sciences Center, Oklahoma City, Oklahoma 73104, USA.

*These authors contributed equally to this work.
} 
Clonal expansions accounted for $43 \%$ of the ASC variable regions from the 14 immunized donors, including three with over $70 \%$ clonality (Fig. 2a). In stark contrast, based on $\mathrm{VH}$ regions sequenced from our laboratory in a comparable fashion ${ }^{15-17}$, naive and memory B cells (IgM or IgG) isolated from blood were rarely or never clonal, whereas for tonsillar B cells only $10 \%$ of IgM and $12 \%$ of IgG germinal centre and memory cells were clonally related.

Immunoglobulin variable gene somatic hypermutation allows for the generation of high-affinity antibodies ${ }^{18,19}$. Surprisingly, the influenza-specific ASCs had accumulated more somatic mutations than any normal population of $\mathrm{B}$ cells. Considering the various donors (Fig. 2b), the ASCs averaged 19.4 $\pm 3.5 \mathrm{VH}$ gene mutations, which is greater than that of germinal centre or memory B cells which average 13.6 \pm 4.8 mutations for IgG or $8.4 \pm 3.8$ mutations for IgM. A surprising 11\% (41/405) of the ASC VH gene segments have more than 30 of 300 (or about $10 \%$ ) of the total nucleotides altered (Fig. 2c). A preference for complementarity-determining region replacement mutations suggests that the ASCs were functionally selected (Supplementary Table 1). These observations suggest the origin of the anti-influenza ASCs is predominantly memory B cells that probably accumulated new mutations on this and on previous rounds of activation.

It is not known how often the ASCs that are induced by vaccination produce high-affinity antibodies against influenza. Immunoglobulin variable region genes from ASCs can be used to express specific antibodies $^{20}$. We therefore used the variable gene transcripts of isolated single ASCs to express recombinant mAbs in the human 293 cell line (Supplementary Fig. 1b and Methods). The ASCs of five donors were isolated at day 7 post-vaccination. From these, 71\% (61/86) of the antibodies bound with high affinity to either native antigens of

a Gated for CD20low/CD3-
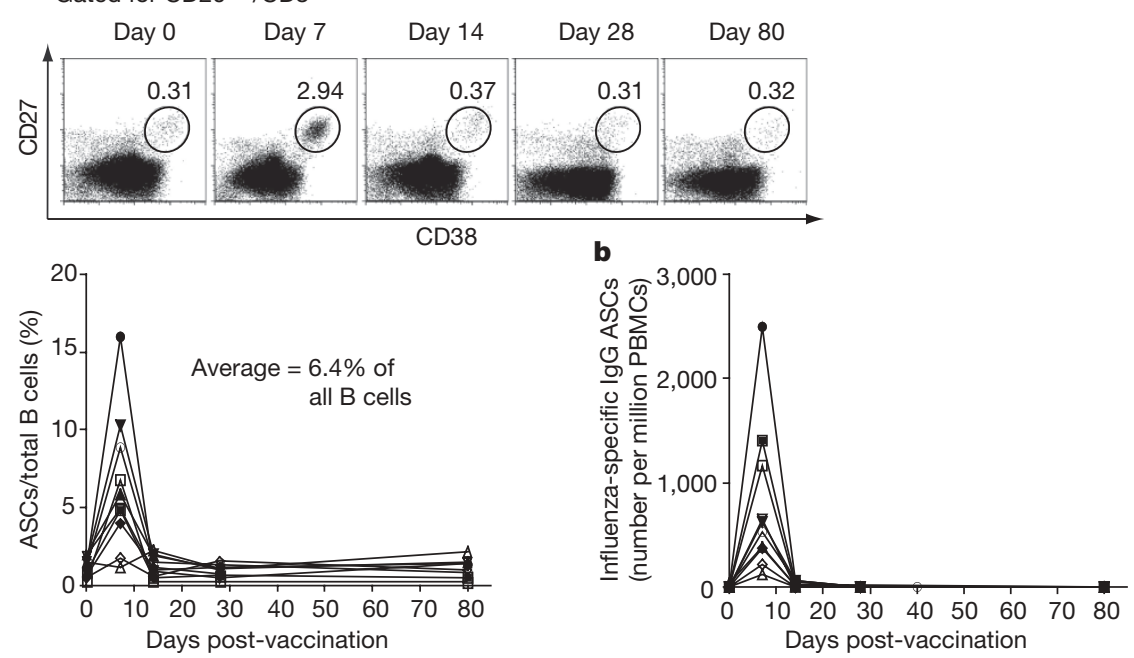

c ASCs were recently generated

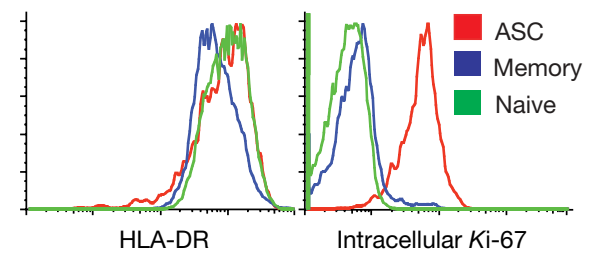

e

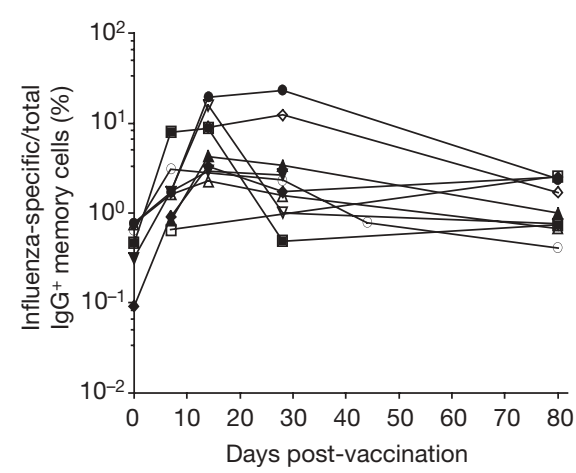

Figure 1 | Analysis of the B-cell response induced by influenza vaccination. a, ASCs were measured in blood by flow cytometry. Shown is the frequency of the ASC gate $\left(\mathrm{CD}^{-} / \mathrm{CD} 20^{- \text {llow }} / \mathrm{CD} 19^{+} / \mathrm{CD} 27^{\mathrm{hi}} / \mathrm{CD} 38^{\mathrm{hi}}\right)$ for a representative donor and a summary for all ten donors normalized to total $\mathrm{CD}^{+} 0^{+} / \mathrm{CD} 19^{+}$B-cell numbers. b, Peripheral blood mononuclear cells (PBMCs) collected from ten donors were assayed for influenza-specific IgG secreting ASCs by ELISPOT assay at 0, 7, 14, 28 and 80 days after vaccination. Each sample was measured in duplicate, averaged and plotted as d Isolating influenza-specific ASCs

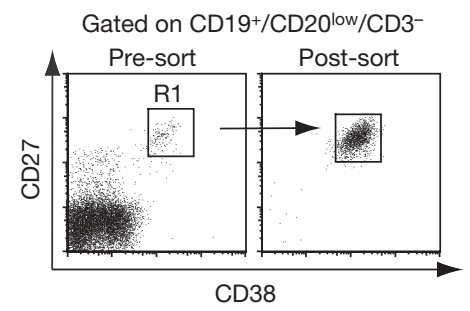

ELISPOT of 50 ASCs (R1 gate)

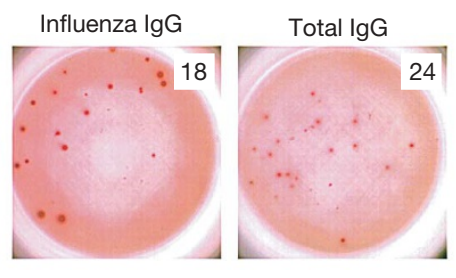

ASCs per million PBMCs over time post-vaccination. c, HLA-DR and intracellular expression of $\mathrm{Ki}-67$ by ASCs compared with naive or memory B cells. d, Most ASCs at day 7 after influenza vaccination are influenza specific. Influenza- and total IgG-specific ELISPOT assays from several donors gave similar results. e, Percentage of influenza-specific memory cells per total IgG-positive memory cells after mitogen stimulation as measured by ELISPOT at $0,7,14,28$ and 80 days post-vaccination as previously described $^{14}$. 
the influenza vaccine strains $(53 / 86,61 \%)$ or to components of the vaccine only (8/86, 9\%) (Fig. 3, Table 1 and Supplementary Fig. 2). We suspect that the epitopes found only in the vaccine are exposed on the fixed virions or are from added preservatives. In comparison, none of the 86 mAbs generated from naive B cells ${ }^{15}$ (Fig. 3b) and only one of 54 antibodies from random IgG memory B cells bound to the influenza vaccine strains with appreciable affinity (data not shown). The antibodies produced from the influenza-specific ASCs bound to any of the three vaccine components with similar frequency (Table 1 and Supplementary Fig. 2). Analysis of viral antigen specificity by immunoprecipitation and western blot (Supplementary Fig. 3) found that $60 \%$ of the influenza-reactive antibodies bound to haemagglutinin, of which half were haemagglutination inhibiting (HAI) (Fig. 3c and Table 1). Twelve per cent of the antibodies bound to neuraminidase or to other minor components of the vaccine likely residual to the purification of haemagglutinin and neuraminidase during vaccine production. Ten per cent of the antibodies did not precipitate native antigens and bound only to epitopes on denatured viral proteins detectable by western blot. Importantly, each of three representative $\mathrm{HAI}^{+}$antibodies against influenza-A (anti-H3N2) and one against influenza-B from the day-7 ASCs (Table 1, bold) were found to neutralize viral infection of Madin-Darby canine kidney (MDCK) cells in vitro (each neutralized virus at less than $1 \mu \mathrm{g} \mathrm{ml}^{-1}$ antibody, Supplementary Fig. 4). In conclusion, after
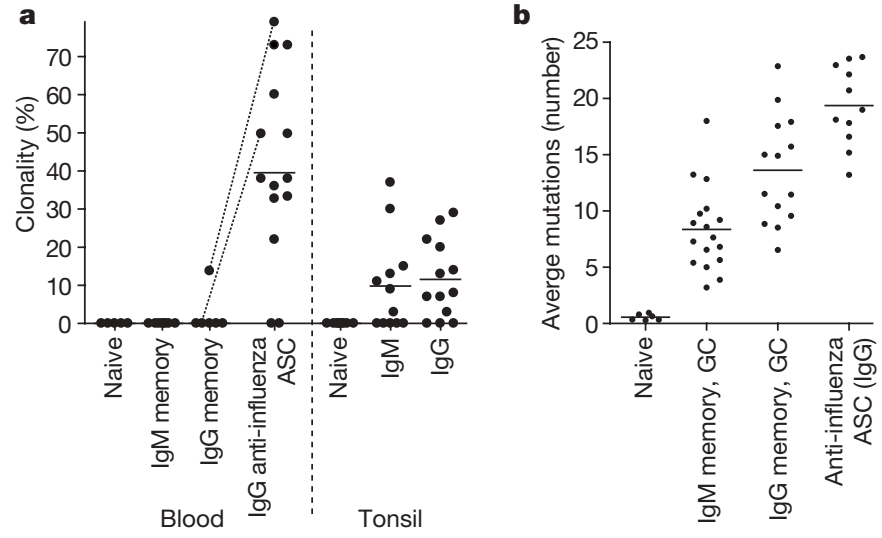

C

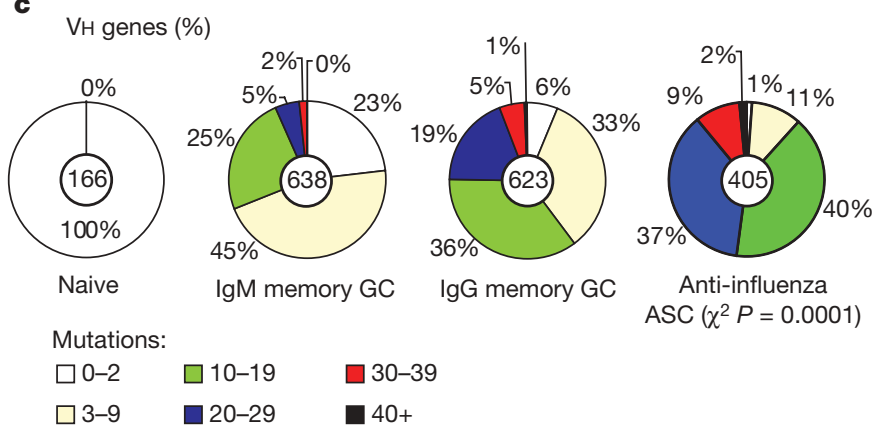

Figure 2 | The ASC response after influenza vaccination is pauci-clonal and highly diversified by somatic hypermutation. a, Comparison of the mean proportion (line) of all clonal variable region sequences from day-7 ASCs of 14 donors (points), including the bulk RNA of $10^{4}-10^{5}$ ASCs from ten donors and verification by single-cell RT-PCR for four donors (average 37 sequences per donor). The ASCs were the most clonally related population ( $t$-test, $P \leq 0.0003)$. Dotted lines indicate donors from which memory and ASCs were analysed simultaneously. Other B-cell populations were from historical data analysed in a similar fashion from our laboratory ${ }^{15-17}$ (see Methods and Supplementary Data) $\mathbf{b}$, Each point is the average frequency of somatic mutations per sequence from each donor ( $n$ values within Methods). On average, the anti-influenza ASCs had accumulated more mutations than either the IgG ( $t$-test $P=0.003)$ or $\operatorname{IgM}(P=<0.0001)$ memory and germinal-centre populations. GC, germinal centre. $c$, The proportion of all variable genes from each B-cell population with the number of somatic mutations denoted in the legend ( $n$ values are at the centre of each pie chart). influenza vaccination, early ASCs produce functional antibodies that bind with high affinity and likely provide early protection.

Although most of the ASCs arise only after vaccination (Fig. la), $29 \%$ of the antibodies generated did not detectably bind to the

a $71 \%(61$ of 86$)$ of lgG ASCs bind influenza virions $(62 \%, 53 / 86)$ or the vaccine only (9\%, 8/86, not shown) $\left(K_{\mathrm{d}}=10^{-8}-10^{-11}\right)$
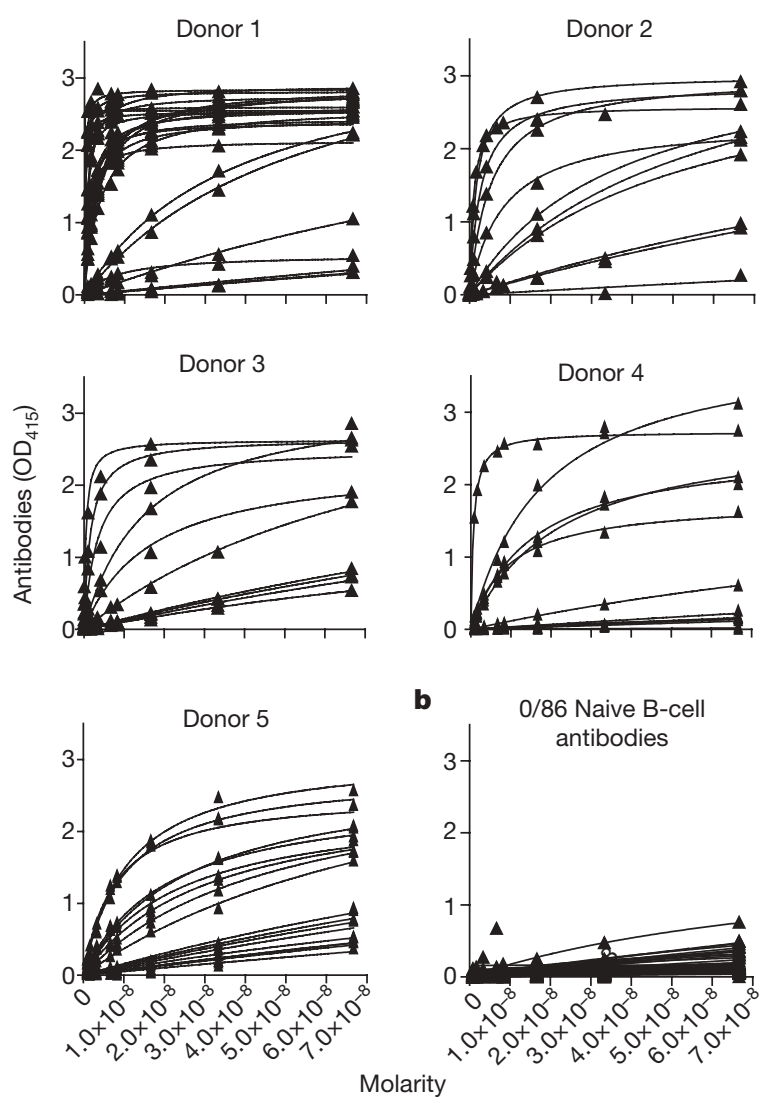

c
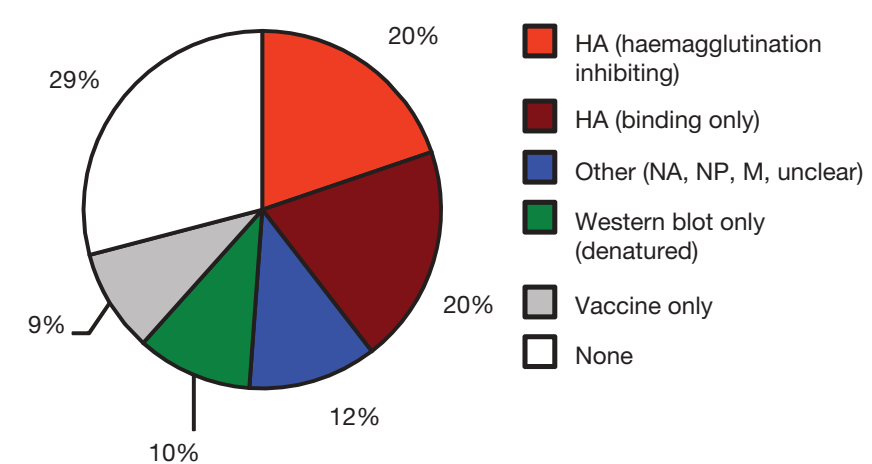

Figure 3 | High-affinity mAbs generated from single influenza-specific ASCs. a, Recombinant mAbs from day-7 IgG anti-influenza ASCs (Supplementary Fig. 1b) bind to a mixture of the three influenza vaccine strain virions with high affinity. In total, $71 \%$ of the ASC antibodies bound either native antigens of influenza viruses freshly grown in eggs $(53 / 86,62 \%)$, or to antigens within the vaccine only (8/86, $9 \%$, not shown). Antibodies from each of the five donors were influenza specific (by donor, 34, 13, 11, 15 and 21 antibodies were generated, of which $45-85 \%$ were influenza specific). Individual antibody strain specificities are shown in Table 1 and

Supplementary Fig. 2. b, None of 86 naive B-cell antibodies bound influenza. c, Analysis by immunoprecipitation and western blot (Supplementary Fig. 3) identified the specific viral antigens bound. Haemagglutination assays identified those antibodies that were inhibiting (Table 1 and Methods). HA, haemagglutinin; NA, neuramininidase; NP, nuclear protein; $M$, matrix protein. 
influenza strains or whole vaccine (Fig. 3c). Possible causes include errors introduced by the reverse-transcription polymerase chain reaction (RT-PCR) steps (though PCR errors were rare, Supplementary Table 2), targeting of non-viral or denatured components of the vaccine or antigens only evident physiologically, bystander activation of non-specific memory cells ${ }^{8}$, or displacement of non-specific plasma cells from the bone marrow ${ }^{13}$. The last possibility is unlikely as expression of HLA-DR ${ }^{13}$ and Ki-67 (Fig. 1c) by the ASCs suggests they were newly generated.

The long-held theory of OAS suggests that new influenza variants will evade surveillance when memory B cells reactive to previous viral strains dominate the response ${ }^{1}$. To consider the impact of OAS directly, we compared the relative affinity with either the current $\mathrm{B}$ strain virus (B/Malaysia/2506/2004) or with the two previous ones (B/Shanghai/361/2002 or B/Hong Kong/33/2001) (Fig. 4a, b). In the 2006/7 season, antibodies were analysed from five donors who had also been vaccinated in the $2005 / 6$ season and one in 1991 , so that

\section{Table $1 \mid$ Characteristics of anti-influenza antibodies}

H1N1: A/New Caledonia/20/99

\begin{tabular}{lllll}
\hline & Antibody & $K_{\mathrm{d}}(\mathrm{M})$ & HAl & Antigen \\
\hline Donor 1 & D1-1 & $2.70 \times 10^{-10}$ & None & Haemagglutinin \\
& D1-2 $(\times 2)^{*}$ & $7.33 \times 10^{-10}$ & None & Haemagglutinin \\
& D1-3 $(\times 2)^{*}$ & $1.55 \times 10^{-9}$ & None & Haemagglutinin \\
& D1-4 & $2.14 \times 10^{-9}$ & None & Haemagglutinin \\
& D1-5 & $2.66 \times 10^{-9}$ & 1 & Haemagglutinin \\
& D1-6 & $3.62 \times 10^{-9}$ & 1 & Haemagglutinin \\
Donor 2 & D2-1 & $2.73 \times 10^{-9}$ & None & 85 kilodalton $(k D a)$ band on \\
& & & & western blot \\
& D2-2 & $5.42 \times 10^{-9}$ & None & 85 kDa band on western blot \\
& D2-3 & $5.20 \times 10^{-9}$ & None & Haemagglutinin \\
Donor 3 & D3-1 & $1.88 \times 10^{-9}$ & None & 85 kDa band on western blot \\
Donor 4 & D4-1 & $4.00 \times 10^{-8}$ & None & 85 kDa band on western blot \\
& D4-2 & $1.65 \times 10^{-8}$ & None & Haemagglutinin \\
Donor 5 & D5-1 & $5.01 \times 10^{-11}$ & None & Multiple bands on western blot \\
& D5-2 & $1.01 \times 10^{-9}$ & None & Denatured haemagglutinin-1 on \\
& & & & western blot \\
& D5-3 & $1.78 \times 10^{-8}$ & None & Haemagglutinin \\
& D5-10 & $1.78 \times 10^{-8}$ & None & Multiple bands on western blot \\
\end{tabular}

H3N2: A/Wisconsin/67/2005(2006/7) or A/California/7/2004 (for donor 3)

\begin{tabular}{lllll}
\hline Donor 1 & D1-7 & $7.72 \times 10^{-11}$ & 128 & Haemagglutinin \\
& D1-8 & $2.86 \times 10^{-10}$ & 4 & Haemagglutinin \\
& D1-9 $(\times \mathbf{4})^{*}$ & $3.77 \times 10^{-10}$ & 8 & Haemagglutinin \\
& D1-10 & $4.18 \times 10^{-10}$ & 4 & Haemagglutinin \\
& D1-11 & $1.57 \times 10^{-9}$ & None & Nuclear protein \\
Donor 2 & D2-4 & $3.62 \times 10^{-10}$ & 2 & Haemagglutinin \\
& D2-5 & $8.29 \times 10^{-9}$ & None & Unclear \\
Donor 3 & D3-2 & $3.50 \times 10^{-9}$ & None & Haemagglutinin \\
& D3-3 & $1.56 \times 10^{-8}$ & None & Haemagglutinin \\
& D3-4 & $4.86 \times 10^{-10}$ & 32 & Haemagglutinin \\
Donor 4 & D4-3 & $4.56 \mathrm{E}-09$ & 1 & Haemagglutinin
\end{tabular}

B strains: B/Malaysia/2506/2004 or B/Shanghai/361/2004 (donors 3 and 6)

\begin{tabular}{lllll}
\hline Donor 1 & D1-12 & $1.93 \times 10^{-10}$ & None & Haemagglutinin \\
& D1-13 & $2.04 \times 10^{-10}$ & 16 & Haemagglutinin \\
& D1-14 $(\times 2) *$ & $2.43 \times 10^{-10}$ & None & Haemagglutinin \\
& D1-15 & $2.47 \times 10^{-10}$ & 8 & Haemagglutinin \\
& D1-16 & $6.20 \times 10^{-10}$ & None & Haemagglutinin \\
& D1-17 & $6.33 \times 10^{-10}$ & None & Haemagglutinin \\
& D1-18 & $4.74 \times 10^{-10}$ & None & Haemagglutinin \\
& D1-19 & $3.70 \times 10^{-8}$ & None & Matrix protein on western blot \\
Donor 2 & D2-6 & $1.71 \times 10^{-9}$ & None & Unclear \\
& D2-7 & $5.77 \times 10^{-8}$ & None & Matrix protein on western blot \\
& D2-8 & $3.66 \times 10^{-8}$ & None & Haemagglutinin \\
Donor 3 3 D-5 & $1.66 \times 10^{-8}$ & None & Unclear (low affinity) \\
& D3-6 & $1.62 \times 10^{-8}$ & None & Denatured nuclear protein on \\
& & & & western blot \\
Donor 4 4 D4-4 & $1.71 \times 10^{-8}$ & 1 & Haemagglutinin \\
& D4-5 & $3.28 \times 10^{-8}$ & 4 & Haemagglutinin \\
Donor 5 5 -5 & $3.78 \times 10^{-8}$ & None & Unclear (low affinity) \\
& D5-6 & $1.11 \times 10^{-8}$ & None & Matrix protein on western blot \\
& D5-7 & $1.26 \times 10^{-8}$ & None & Unclear (low affinity) \\
& D5-8 & $4.8 \times 10^{-8}$ & None & 85 kDa band on western blot \\
Donor 6 6 D-1 & $5.04 \times 10^{-10}$ & 4 & Haemagglutinin \\
\hline
\end{tabular}

*Clonal expansions with number of clones indicated; bold, mAbs tested for viral neutralization.

reactive memory cells should have been readily available for an OAS response. Importantly, each of the 19 anti-B strain antibodies bound to the new B strain with equal, and in most cases with greater, affinity than the previous vaccine strains (Fig. 4c and Supplementary Fig. 2). This adaptation occurred despite the $10 \%$ or less difference of the

a

1988-89 B/Victoria/2/87

1989-91 B/Yamagata/16/88

1991-95 B/Panama/45/90

1995-01 B/Beijing/184/93

2001-02 B/Sichuan/379/99

2002-04 B/Hong Kong/330/2001

2004-06 B/Shanghai/361/2002

2006-07 B/Malaysia/2506/04 b

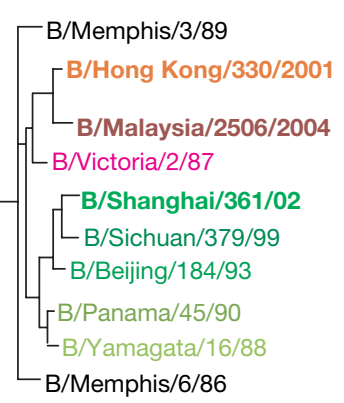

C 2006-07 vaccine:
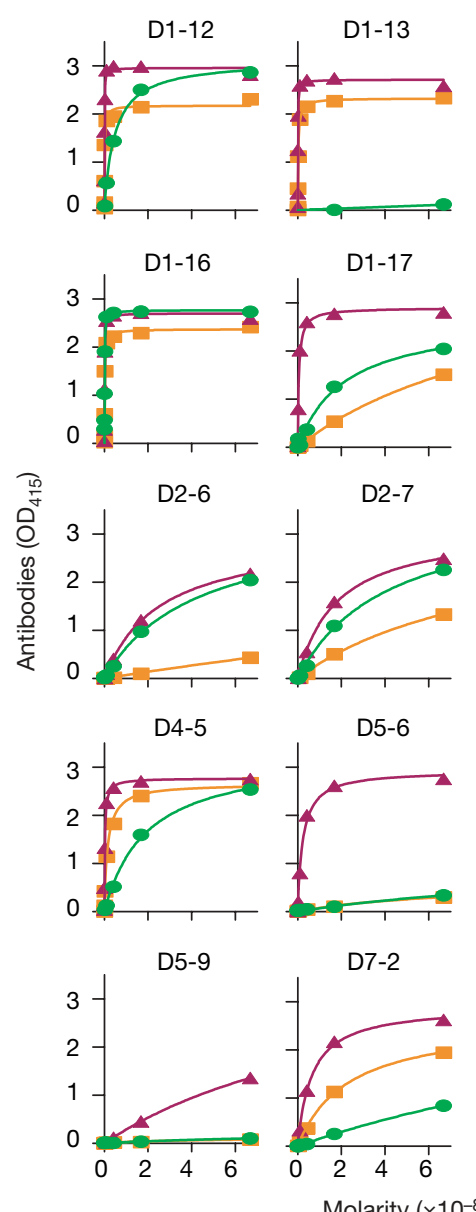

D7-2

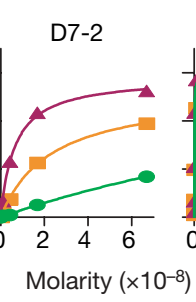

2002-04 B/Hong Kong/330/2001

2004-06 B/Shanghai/361/02

2006-07 B/Malaysia/2506/2004
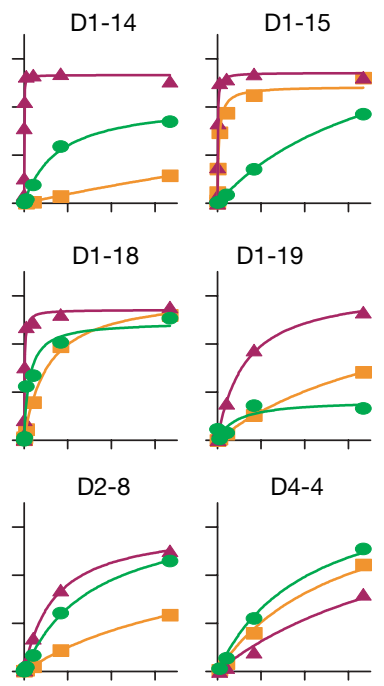

D4-4

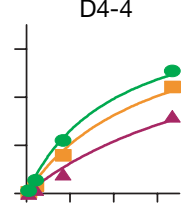

D5-7

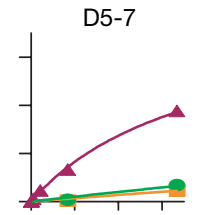

D5-8

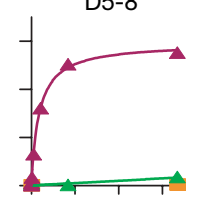

D9-1

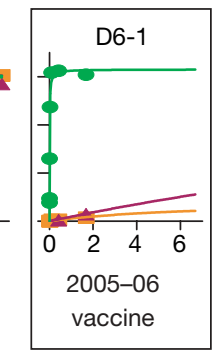

\section{$\triangle$}

Figure 4 | Specificity for the newly introduced influenza-B strain in the vaccine suggests a minimal impact of OAS. a, Influenza-B strains used for the vaccine since 1989. Throughout the figure, strain names are colour coded for the Yamagata lineage (green) and the Victoria lineage (orange/red).

b, Phylogenetic tree illustrating the similarity of recent influenza-B strains and the years in which each strain was included in the vaccine. The three vaccine strains tested (bold font) included B/Malaysia/2506/2004 (2006/7 season), which is most similar to the 2002-2004 strain (B/Hong Kong/33/ 2001 ). Conversely, the $2005 / 6$ vaccine strain, $B /$ Shanghai $/ 361 / 2002$, is more divergent. c, All anti-B-strain antibodies reacted with equal or greater affinity to the current year's vaccines when tested by ELISA. 
haemagglutinin sequence of the 2006/7 B strain from those used in previous vaccines. Although previous exposure to B/Malaysia/2506/ 2004 cannot be entirely excluded, there was no history of exposure, and pre-vaccination serum titres of antibody against B/Malaysia/ 2506/2004 were not above background levels (data not shown). Thus we conclude that even for the earliest detectable influenzaspecific B cells after vaccination, the ASCs, OAS does not limit reactivity to newly introduced influenza strains.

In conclusion, we show that after influenza vaccination we can isolate an almost entirely antigen-specific population of ASCs that comprise about $5 \%$ of all blood-borne B cells. Further, our findings help to resolve a major, long-standing obstacle in the field of medicine $^{21}$ : the rapid production of fully human mAbs. Antibody or serum therapy has been demonstrated to treat a plethora of diseases effectively, but it is not widely used because sometimes fatal anaphylactic responses and serum sickness are common. These obstacles can only be overcome by using fully human mAbs. Our findings demonstrate that we can now generate human mAbs from the antigen-specific ASCs directly, and within only weeks of vaccination (Supplementary Fig. 1c). With a modern resurgence of interest in monoclonal antibody therapy, we anticipate that antibodies produced from post-vaccination ASCs will generate substantial advances for the treatment of infectious diseases.

Conventional wisdom holds that the level of pre-formed antibody is the main correlate of protection against influenza virus. However, our results, showing the rapidity of the antibody response after vaccination and the high affinity of the antibodies produced, strongly suggest that the recall response could also play a role in protective immunity. This antibody would not, of course, prevent initial infection but could play a crucial role in preventing the spread of virus and bringing about faster resolution of the infection. This notion is supported by our finding that OAS was not a significant aspect of the memory response, as the antibodies produced were highly specific to the immunizing antigen.

\section{METHODS SUMMARY}

Detailed methods are found in the Methods and Supplementary Information. Healthy volunteers received either the Fluzone 2005-06 (Aventis Pasteur Inc) or Fluvirin 2006-07 (Chiron) influenza vaccine formulations. This project had institutional review board approvals from both Emory University School of Medicine and the Oklahoma Medical Research Foundation. Enumeration of IgG or influenza-specific B cells has been previously described, including: ELISPOT $^{22}$ and polyclonal activation of memory B cells ${ }^{14,22}$. HAI titres and viral neutralization were determined by standard procedures ${ }^{23}$. Monoclonal antibodies were tested for $\mathrm{HAI}$ at $30 \mu \mathrm{g} \mathrm{ml}^{-1}$ and five twofold serial dilutions. The ASCs were identified as $\mathrm{CD}^{-} / \mathrm{CD} 20^{-/ \text {low }} / \mathrm{CD} 19^{+} / \mathrm{CD} 27^{\text {hi }} / \mathrm{CD} 38^{\text {hi }}$ cells. Isolation of other B-cell types and methods for RT-PCR of the variable genes were as previously described ${ }^{15,17}$. An average of 35 variable gene sequences was analysed from each donor (Methods and Supplementary Information). The leader and constant regions that are rarely targeted by somatic mutation were primed for RT and PCR to avoid biases between populations for the variable gene repertoire and clonality determinations. The single-cell RT-PCR methods and the procedures for production of recombinant mAbs were as previously described ${ }^{15,24}$. Monoclonal antibodies were screened against fresh influenza virions grown in chicken eggs. Antibody affinities $\left(K_{\mathrm{d}}\right)$ were calculated by nonlinear regression (GraphPad Prism software) of influenza enzyme-linked immunosorbent assay (ELISA) curves plotted from eight dilutions of antibody ranging from $10 \mu \mathrm{g} \mathrm{ml}^{-1}$ to $0.125 \mu \mathrm{g} \mathrm{ml}^{-1}$.

Full Methods and any associated references are available in the online version of the paper at www.nature.com/nature.

\section{Received 16 October 2007; accepted 4 March 2008.} Published online 30 April 2008.

1. Francis, T. Jr. On the doctrine of original antigenic sin. Proc. Am. Phil. Soc. 104, 572-578 (1960).
2. Ahmed, R., Oldstone, M. B. \& Palese, P. Protective immunity and susceptibility to infectious diseases: lessons from the 1918 influenza pandemic. Nature Immunol. 8, 1188-1193 (2007).

3. Subbarao, K. \& Joseph, T. Scientific barriers to developing vaccines against avian influenza viruses. Nature Rev. Immunol. 7, 267-278 (2007).

4. Gerhard, W. et al. Role of the B-cell response in recovery of mice from primary influenza virus infection. Immunol. Rev. 159, 95-103 (1997).

5. Luke, T. C., Kilbane, E. M., Jackson, J. L. \& Hoffman, S. L. Meta-analysis: convalescent blood products for Spanish influenza pneumonia: a future H5N1 treatment? Ann. Intern. Med. 145, 599-609 (2006).

6. Puck, J. M., Glezen, W. P., Frank, A. L. \& Six, H. R. Protection of infants from infection with influenza $A$ virus by transplacentally acquired antibody. J. Infect. Dis. 142, 844-849 (1980).

7. Simmons, C. P. et al. Prophylactic and therapeutic efficacy of human monoclonal antibodies against H5N1 influenza. PLoS Med. 4, e178 (2007).

8. Bernasconi, N. L., Traggiai, E. \& Lanzavecchia, A. Maintenance of serological memory by polyclonal activation of human memory B cells. Science 298 2199-2202 (2002)

9. Brokstad, K. A. et al. Parenteral influenza vaccination induces a rapid systemic and local immune response. J. Infect. Dis. 171, 198-203 (1995).

10. Brokstad, K. A. et al. Parenteral vaccination against influenza does not induce a local antigen-specific immune response in the nasal mucosa. J. Infect. Dis. 185, 878-884 (2002)

11. Sasaki, S. et al. Comparison of the influenza virus-specific effector and memory B-cell responses to immunization of children and adults with live attenuated or inactivated influenza virus vaccines. J. Virol. 81, 215-228 (2007).

12. Poulsen, T. R. et al. Kinetic, affinity, and diversity limits of human polyclonal antibody responses against tetanus toxoid. J. Immunol. 179, 3841-3850 (2007).

13. Odendahl, M. et al. Generation of migratory antigen-specific plasma blasts and mobilization of resident plasma cells in a secondary immune response. Blood 105, 1614-1621 (2005).

14. Crotty, S., Aubert, R. D., Glidewell, J. \& Ahmed, R. Tracking human antigenspecific memory B cells: a sensitive and generalized ELISPOT system. J. Immunol. Methods 286, 111-122 (2004).

15. Koelsch, K. et al. Mature B cells class switched to $\lg D$ are autoreactive in healthy individuals. J. Clin. Invest. 117, 1558-1565 (2007).

16. Zheng, N. Y., Wilson, K., Jared, M. \& Wilson, P. C. Intricate targeting of immunoglobulin somatic hypermutation maximizes the efficiency of affinity maturation. J. Exp. Med. 201, 1467-1478 (2005).

17. Zheng, N. Y. et al. Human immunoglobulin selection associated with class switch and possible tolerogenic origins for $\mathrm{C}$ delta class-switched B cells. J. Clin. Invest. $113,1188-1201$ (2004).

18. Clarke, S. H. et al. Inter- and intraclonal diversity in the antibody response to influenza hemagglutinin. J. Exp. Med. 161, 687-704 (1985).

19. Cook, W. D. \& Scharff, M. D. Antigen-binding mutants of mouse myeloma cells. Proc. Natl Acad. Sci. USA 74, 5687-5691 (1977).

20. Meijer, P. J. et al. Isolation of human antibody repertoires with preservation of the natural heavy and light chain pairing. J. Mol. Biol. 358, 764-772 (2006).

21. Llewelyn, M. B., Hawkins, R. E. \& Russell, S. J. Discovery of antibodies. Br. Med. J. 305, 1269-1272 (1992).

22. Crotty, S. et al. SAP is required for generating long-term humoral immunity. Nature 421, 282-287 (2003).

23. Webster, R., Cox, N. \& Stohr, K. World Health Organization Manual on Animal Influenza Diagnosis and Surveillance (WHO, Geneva, 2002).

24. Wardemann, H. et al. Predominant autoantibody production by early human B cell precursors. Science 301, 1374-1377 (2003).

Supplementary Information is linked to the online version of the paper at wwW.nature.com/nature.

Acknowledgements We thank A. Popkowski, H. Wu, L. Abraham and B. Begley for technical assistance, and R. Casellas and J. Knight for reading the manuscript. This work was funded in parts by National Institutes of Health $(\mathrm{NIH})$ grant numbers HHSN266200500026C (P.C.W.), P20 RR018758 (P.C.W.), NIH/National Institute of Allergy and Infectious Diseases (NIAID) U19-AI057266-04 (R.A.), NIH/NIAID HHSN266200700006C Center of Excellence for Influenza Research and Surveillance (R.A.) and NIH/NIAID N01-AI-50025-02 (R.A. and C.L.). J.W. was supported by a postdoctoral fellowship from The Swedish Research Council.

Author Information Reprints and permissions information is available at www.nature.com/reprints. Correspondence and requests for materials should be addressed to P.C.W. (wilsonp@omrf.org). 


\section{METHODS}

Cell and serum isolation. All studies were pre-approved by the institutional review boards of Emory University School of Medicine and the Oklahoma Medical Research Foundation. Healthy volunteers received influenza vaccine formulations (Fluzone, Aventis Pasteur, 2005/6, or Fluvirin, Chiron, 2006/7). PBMCs were isolated using Vacutainer tubes (Becton Dickinson) or lymphoprep gradient (CellGro), washed and re-suspended in supplemented culture media or PBS. Plasma was heat inactivated.

ELISPOT and memory B-cell assays. ELISPOT and memory assays were as previously described ${ }^{14,25}$ Total IgG secreting or influenza-specific ASCs were detected using 1/20 diluted influenza vaccine in PBS (as above) or with goat anti-human Ig (Caltag). Dilutions of washed PBMCs incubated in supplemented RPMI medium for $2 \mathrm{~h}$ were incubated in ELISPOT plates for $6 \mathrm{~h}$. After washing the plates, ASC antibody was detected with anti-huIgG-biotin (Caltag) and avidin-D-HRP (Vector Laboratories) and developed with AEC substrate (Sigma) before analysis on an ELISPOT counter (Cellular Technologies Ltd.). Memory cells were detected by incubating PBMCs at $5 \times 10^{5}$ cells per millilitre in R-10 supplemented with pokeweed mitogen extract, phosphothiolated CpG ODN-2006 $6^{26}$ and Staphylococcus Aureus Cowan (Sigma). After culture for six days, the cells were washed and quantified by ELISPOT.

Flow cytometry and cell sorting. Flow cytometry was performed on whole blood after lysis of erythrocytes. Mostly Pharmingen antibodies were used for quantifying ASC or memory cells (Fig. 1) except anti-CD27-APC (ebiosciences) and goat anti-huIgG-FITC (Southern Biotechnologies). For single-cell analysis and production of mAbs, antibodies used included anti-CD3-FITC, anti-CD20FITC, anti-CD38-APC-Cy5.5, anti-CD27-PE, anti-IgG-Alexa-647 and antiCD19-PE-Alexa 610 from Caltag, plus anti-IgD-biotin and strepavidin-Pe-Cy7 (Pharmingen). ASCs were gated as $\mathrm{IgG}^{+} / \mathrm{IgD}^{-} / \mathrm{CD} 9^{+} / \mathrm{CD}^{-} / \mathrm{CD} 20^{\text {low }} /$ $\mathrm{CD} 27^{\text {high }} / \mathrm{CD} 38^{\text {high }}$. All other cell types were isolated as previously described ${ }^{15-17}$. Cytometry data was analysed using FlowJo software.

Single-cell RT-PCR and PCR of antibody variable region genes. As detailed in Supplementary Information, single B cells were sorted into 96-well PCR plates containing RNase inhibitor (Promega). $\mathrm{VH}$ and $\mathrm{V \kappa}$ genes from each cell were amplified by RT-PCR and nested PCR reactions using cocktails of primers as previously described ${ }^{15,24}$, then sequenced. To generate recombinant antibodies, restriction sites were incorporated by PCR with primers to the particular variable and junctional genes. RT-PCR of bulk RNA to analyse V genes was as previously described ${ }^{15,17,27}$.

Analysis of clonality and somatic mutations of variable region genes. To quantify clonality, variable genes were randomly sequenced from the bulk RNA of ASCs from ten donors (by donor, $n=22,47,49,12,16,19,36,25,34$ and 63) and verified by single-cell RT-PCR analysis of ASCs from four donors $(n=65,37,30$ and 50). Naive, memory and germinal-centre cell variable gene libraries included the following VH gene $n$ values: blood naive (by donor, $n=61$, $24,15,14$ and 24), blood IgM memory $(n=28,17,27,11,23,12,29$ and 20), blood IgG memory ( $n=23,18,18,17,22$ and 21$)$, tonsillar naive B cells ( $n=125$, $32,16,22,32,23,46$ and 81$)$, tonsillar IgM and germinal centre/memory $(n=50$, $42,35,16,60,15,50,25,39,19,55$ and $58 \mathrm{VH}$ genes) and tonsillar IgG germinal centre/memory $(n=113,25,14,40,12,41,11,23,18,51,15,54$ and 69$)$. The $n$ values for analysis of somatic hypermutation included: anti-influenza ASCs from 11 donors $(n=63,18,33,46,49,11,36,11,30,35,25)$; IgG germinal centre/ memory cells from 14 donors ( $n=110,37,19,28,174,40,25,15,21,18,22,24$, $19,71)$; IgM germinal centre/memory from 17 donors $(n=56,158,18,91,17$, $10,16,30,19,28,11,36,29,13,22,20,64)$; and naive cells from six donors $(n=18,42,21,34,15,36)$. Background mutation rates were insignificant (Supplementary Table 2).

Recombinant monoclonal antibody expression and analysis. All assays are further detailed in the Supplementary Information. VH or Vк genes amplified from each single cell were cloned into IgG1 or Igא expression vectors as previously described ${ }^{15,24}$. Heavy- and light-chain plasmids were co-transfected into the 293A cell line for expression, and antibodies purified with protein A sepharose. The influenza virus strains used for ELISA or HAI were freshly grown in eggs and purified by standard methods ${ }^{23}$ and included: A/New Caledonia/20/9 (H1N1), A/California/7/2004 (H3N2) for 2005/6 or A/Wisconsin/67/2005 (H3N2) for 2006/7, and B/Shanghai/361/2002-like for 2006/2007or B/Malaysia/ $2506 / 2004$ for $2006 / 7$. Serum or mAb HAI titres were determined as previously described $^{28}$. After ELISA screening with a cocktail of all influenza strains and 1/20 dilutions of the vaccines, the affinity and specificity of binding-positive mAbs were determined with the individual influenza viruses. ELISA affinities were calculated by nonlinear regression analysis of curves from eight dilutions of antibody $\left(10-0.125 \mu \mathrm{g} \mathrm{ml}^{-1}\right)$ using GraphPad Prism. Influenza-neutralizing activity was detected as inhibition of MDCK cell death by $50 \%$ tissue culture infectious doses of A/Wisconsin/67/2005 or B/Shanghai/361/2002 based on the protocol of the World Health Organization manual23.

Immunoprecipitation and western blot analyses. All assays are further detailed in the Supplementary Information. For immunoprecipitation, 8 haemagglutinin units (HAU) of virus were lysed and incubated with $10 \mu \mathrm{g} \mathrm{ml}^{-1}$ of mAb before purification with Protein A-Sepharose (Pierce). mAb was eluted from the protein-A by boiling in Laemmli buffer (Bio-Rad) and analysed on $12 \%$ Tris-glycine polyacrylamide gels. Protein was detected by staining the gels with sypro-orange ( $1 \times$, Invitrogen). For western blots, $8 \mathrm{HAU}$ of virus was diluted and boiled in denaturing/reducing sample buffer, then run on denaturing polyacrylamide gels (as above) followed by electrophoretic transfer to nitrocellulose membranes. The membranes were incubated with each antibody at $5 \mu \mathrm{g} \mathrm{ml}{ }^{-1}$, detected with HRP anti-human IgG (Jackson Immunoresearch) and developed with ECL plus reagent (GE Healthcare). IP gels and western blot membranes were analysed using a STORM840 system (Molecular Dynamics).

Statistics. Statistical analyses (described in context) were performed using GraphPad Prism: frequencies of clonal relatedness and somatic mutation were compared by non-paired, two-tailed Student's $t$-tests; $\chi^{2}$ tests compared summed mutation frequencies.

25. Crotty, S. et al. Cutting edge: long-term B cell memory in humans after smallpox vaccination. J. Immunol. 171, 4969-4973 (2003).

26. Hartmann, G. et al. Delineation of a CpG phosphorothioate oligodeoxynucleotide for activating primate immune responses in vitro and in vivo. J. Immunol. 164, 1617-1624 (2000).

27. Wilson, P. C. et al. Receptor revision of immunoglobulin heavy chain variable region genes in normal human B lymphocytes. J. Exp. Med. 191, 1881-1894 (2000).

28. Stephenson, I. et al. Detection of anti-H5 responses in human sera by $\mathrm{HI}$ using horse erythrocytes following MF59-adjuvanted influenza A/Duck/Singapore/97 vaccine. Virus Res. 103, 91-95 (2004). 